Article

\title{
Finite Element Implementation of a Temperature- Dependent Cyclic Plastic Model for SA508-3 Steel
}

\author{
Jun Tian ${ }^{1}$, Jian Li ${ }^{2}$, Hai Xie ${ }^{1}$, Yu Yang ${ }^{1}$ and Qianhua Kan ${ }^{2, *}$ \\ 1 Science and Technology on Reactor System Design Technology Laboratory, Nuclear Power Institute of China, \\ Chengdu 610213, China; tianjunhi@gmail.com (J.T.); kareemxh@gmail.com (H.X.); tianjunhd@163.com (Y.Y.) \\ 2 Applied Mechanics and Structure Safety Key Laboratory of Sichuan Province, School of Mechanics and \\ Engineering, Southwest Jiaotong University, Chengdu 610213, China; lijian12306@foxmail.com \\ * Correspondence: qianhuakan@home.swjtu.edu.cn; Tel.: +86-028-8760-1442
}

Received: 22 October 2018; Accepted: 14 November 2018; Published: 16 November 2018

check for updates

\begin{abstract}
A new temperature-dependent cyclic plastic model, combining the nonlinear cyclic softening and kinematic hardening rules is established for a nuclear material of SA508-3 steel. A modified isotropic hardening rule is proposed to capture the temperature-dependent cyclic softening, and a modified kinematic hardening rule is established to improve the prediction of the ratcheting behavior by introducing an exponential function related to the accumulated plastic strain. The stress is updated by the radial return mapping algorithm based on the backward Euler integration. A new expression of consistent tangent modulus for the equilibrium iteration is derived, and then the proposed model is implemented into the finite element software ABAQUS by using the user material subroutine (UMAT) to simulate the temperature-dependent ratcheting behaviors of SA508-3 steel. Finally, the ratcheting evolutions of notched bars at elevated temperature are obtained by uniaxial stress-controlled cyclic tests, and the nonuniform strain fields on the surface of plates with a center hole is measured by using the digital image correlation (DIC) technology. Comparisons between experimental and simulated results of a material point and structural examples show that the implemented model can provide reasonable predictions for ratcheting behaviors and nonuniform strain fields of structures at different temperatures for SA508-3 steel.
\end{abstract}

Keywords: SA508-3 steel; ratcheting behavior; cyclic plastic model; temperature-dependence; finite element implementation

\section{Introduction}

Ratcheting is a progressively accumulated inelastic strain under cyclic stressing with a non-zero mean stress. In the process of life prediction and safety assessment, both ASME and RCC-M codes specify that the ratcheting deformation occurring in structural components of nuclear reactor must be addressed. Numerous experiments were carried out to reveal the evolutions of ratcheting behaviors and many nonlinear kinematic hardening models were established to predict ratcheting deformation at different loading conditions [1-4]. Commercial finite element software, such as ANSYS and ABAQUS, are widely applied in engineering design and analysis. The nonlinear kinematic hardening rule, such as the Chaboche's rule [5], is available in these commercial software tools, which can capture the cyclic plastic deformation of some metal materials. For example, a Chaboche-type material model was applied in the Low-Cycle Fatigue finite element assessment on 316L pipes for the "Wendelstein 7-X" stellarator-type nuclear fusion experiment [6], but it commonly gives a conservative prediction [7].

To reasonably describe ratcheting behaviors of different materials, suitable modifications in the kinematic hardening rule are necessary. For example, the material parameter $m_{i}$ in the Ohno-Wang II kinematic hardening rule $[8,9]$ was modified as a function of the maximum equivalent stress 
to improve simulation for ratcheting behaviors of AZ31B magnesium alloy by Lin et al. [10]. To improve the predictions of plastic shakedown and ratcheting behavior of 7075-T6 aluminum alloy, one of three nonlinear backstress components was replaced as a linear term based on the superposed Armstrong-Frederick rule [11]. Besides, Chen et al. [12] proposed a unified viscoplastic model to simulate the time-dependent ratcheting behavior and low cycle fatigue of 316L stainless steel, and provide satisfactory predictions for most experimental results. Focusing on a wide range of heat treatments, the Kocks-Mecking-Estrin model [13] was adopted to predict the cyclic deformation behavior and residual stress of 690 nickel alloy during and after welding.

It must be noted that different materials exhibit different ratcheting behaviors [14]. SA508-3 steel, one kind of nuclear material, is widely used in the nuclear reactor pressure vessel (RPV) of the pressurized water reactor (PWR). The RPV is a piece of key equipment for the first loop of the nuclear plant, of which the structural integrity directly affects the service life of the whole plant. The working temperature of the pressure vessel varies from $25^{\circ} \mathrm{C}$ to $350^{\circ} \mathrm{C}$, and the cyclic thermal and mechanical loadings always run through the whole lifetime. Besides, SA508-3 steel is one of the alloyed ferritic steels. Notable cyclic softening at elevated temperature was observed in the experiments of alloyed ferritic steels, which is totally different from the characteristics of carbon steels and austenitic stainless steels [15]. Therefore, it is worthwhile to develop the temperature-dependent cyclic plastic model for the nuclear material of SA508-3 steel.

Moreover, one of main purposes to develop cyclic plastic models is to simulate the accumulations of plastic strain of structural components under cyclic loadings by finite element method (FEM), which is a foundation to understand the fatigue failure of engineering structures. Therefore, it is necessary to implement these advanced cyclic plastic models into finite element codes. In the process of finite element implementation, the following two problems should be highlighted: one is to determine the stress integration algorithm at each Gauss integration point, the other is to derive the consistent tangent modulus for the equilibrium iteration of finite element analysis. Many progresses have been made to implement cyclic plastic models into commercial finite element softwares, such as ANSYS, ABAQUS, and so on. For example, based on the Abdel Karim-Ohno kinematic hardening rule [16], a cyclic plastic model with a memory surface was implemented into the finite element code ANSYS by using the UMAT [17], which provided a good prediction of the additional hardening caused by the non-proportional loading path. Considering the true stress instead of the effective stress in the yield function, the damage-coupled cyclic plastic model proposed by Bonora [18] was implemented into the finite element code, and an implicit method with an explicit update was used to solve the system of non-linear equations instead of a matrix inversion. An optimization by successive iterations was applied to obtain optimal parameters of the Chaboche model, and the cyclic deformations of nuclear installations [19] made of 316L-N, 316L, and 304L, were successfully simulated. Besides, a multi-objective genetic algorithm was also applied to optimize the parameters of the Chaboche model for 7075-T6 aluminum alloy [20].

Motivated by the above considerations, the present work aims to implement a temperature-dependent cyclic plastic model into finite element software ABAQUS by using the UMAT for SA508-3 steel. The organization of this work is below: firstly, a brief introduction on the Chaboche model is presented. Then, based on the Chaboche model, the discrete material constants are modified into a continuous function corresponding to the accumulated plastic strain. The finite element implementation of the modified model is addressed in detail, including the discretization of constitutive equations, implicit stress integration and derivation of consistent tangent modulus. Finally, finite element models of structures including a notched bar and a plate with a center hole are established, and simulations are carried out to verify the implementation of the present temperature-dependent cyclic plastic model. 


\section{Temperature-Dependent Cyclic Plastic Model}

Considering the temperature term and on the basis of the plastic flow rule at small deformation as well as the initial isotropic elasticity, the total strain rate $\dot{\varepsilon}$ can be written as the sum of three parts, i.e., the elastic strain rate $\dot{\varepsilon}^{e}$, plastic strain rate $\dot{\varepsilon}^{p}$ and thermal strain rate $\dot{\varepsilon}^{T}$.

$$
\dot{\varepsilon}=\dot{\varepsilon}^{e}+\dot{\varepsilon}^{p}+\dot{\varepsilon}^{T}
$$

According to the Hooke's law, the elastic constitutive relation can be expressed as following:

$$
\dot{\boldsymbol{\varepsilon}}^{e}=\mathbf{D}(T)^{-1}: \dot{\boldsymbol{\sigma}}
$$

where $\mathbf{D}(T)$ denotes the temperature-dependent fourth-order elastic tensor, $\dot{\boldsymbol{\sigma}}$ is the stress rate tensor.

The plastic strain rate is formulated based on the flow rule as:

$$
\dot{\varepsilon}^{p}=\sqrt{\frac{3}{2}} \dot{p} \frac{\mathbf{s}-\alpha}{\|\mathbf{s}-\alpha\|}
$$

where $\dot{p}$ is the accumulated plastic strain rate, $\mathbf{s}$ and $\alpha$ represent the deviatoric stress and backstress tensors, respectively. ||| denotes the norm.

The thermal strain rate is defined as:

$$
\dot{\varepsilon}^{T}=\alpha 1 \dot{T}
$$

where $\alpha$ is the thermal expansion coefficient and $\dot{T}$ is the rate of temperature. 1 is the second-order unit tensor.

The von Mises yield surface can be written in the general form:

$$
F_{y}=\sqrt{\frac{3}{2}(\mathbf{s}-\alpha):(\mathbf{s}-\boldsymbol{\alpha})}-Q
$$

where $Q$ represents the isotropic deformation resistance and reflects the change in the radius of yield surface.

The nonlinear kinematic hardening rule revealing the evolution of backstress makes it possible to describe the progressive ratcheting deformation. Based on the Armstrong-Frederick's rule [21], Chaboche [5] firstly decomposed the backstress of into several components, which can be expressed as:

$$
\begin{gathered}
\alpha=\sum_{i=1}^{M} \alpha_{i} \\
\dot{\alpha}_{i}=\frac{2}{3} C_{i} \dot{\varepsilon}^{p}-r_{i} \boldsymbol{\alpha}_{i} \dot{p}
\end{gathered}
$$

where $C_{i}$ and $r_{i}$ are material constants and $M$ is the number of decomposed backstress components. If the number of components increases, a higher prediction accuracy in the accumulation of plastic strain will be obtained, that is, the curve of stress versus plastic strain will be correspondingly divided into more parts (as shown in Figure 1), but it would cost greater computational consumption.

It is noted that the plastic hardening moduli $C_{i}$ can be calculated by differentiating the curve of stress versus accumulated plastic strain with fixed $r_{i}$. Figure 2 shows an exponential function of the plastic hardening modulus $C$ with respect to the accumulated plastic strain at $250{ }^{\circ} \mathrm{C}$ and $300{ }^{\circ} \mathrm{C}$, respectively. 


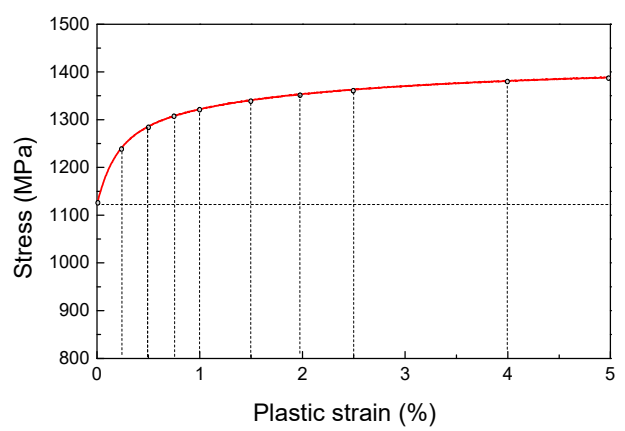

Figure 1. Curves of stress versus plastic strain.

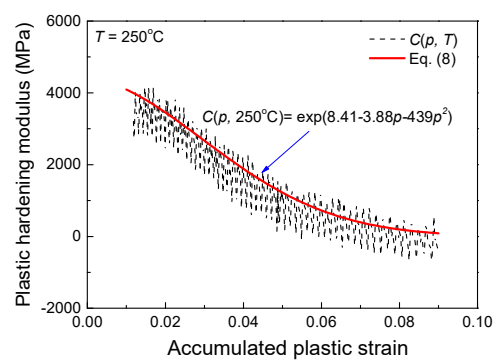

(a)

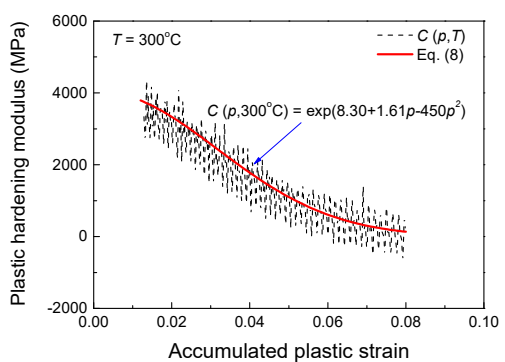

(b)

Figure 2. Exponential curves between the plastic hardening modulus $C(p, T)$ and accumulated plastic strain $p$ at: (a) $250{ }^{\circ} \mathrm{C}$ and (b) $300{ }^{\circ} \mathrm{C}$.

Therefore, an exponential relationship can be used to describe the dependence of the plastic hardening moduli $C_{i}$ on the accumulated plastic strain and temperature as:

$$
C(p, T)=\exp \left(a(T)+b(T) p+c(T) p^{2}\right)
$$

where $a, b$ and $c$ are material constants and can be determined by fitting the corresponding experimental results. Therefore, the evolution of backstress in the kinematic hardening rule can be modified as:

$$
\dot{\alpha}(T)=\frac{2}{3} C(p, T) \dot{\varepsilon}^{p}-r(T) \alpha(T) \dot{p}
$$

where $C(p, T)$ represents the function of the accumulated plastic strain $p$ and the temperature $T$, and $r(T)$ is only relevant to $T$.

Considering the temperature dependence of the isotropic hardening, the temperature-dependent isotropic deformation resistance $Q$ is expressed as:

$$
\dot{Q}(p, T)=\gamma(T)\left(Q_{s a}(T)-Q(p, T)\right) \dot{p}+\frac{\partial Q(p, T)}{\partial T} \dot{T}
$$

where $\gamma(T)$ is a material parameter controlling the rate of resistance $\dot{Q}(p, T) ; Q_{s a}(T)$ represents the saturated isotropic deformation resistance only related to temperature. It should be noted that, when the accumulated plastic strain equals to zero, the initial deformation resistance $Q_{0}$ can be determined from Equation (10). For cyclic softening or cyclic hardening materials, it is observed that the initial deformation resistance $Q_{0}$ in tensile tests is apparently different from that in the subsequent cycles, so the initial value of $Q_{0}$ should be changed at the end of tensile curve in the first cycle. 


\section{Finite Element Implementation}

The cyclic plastic model proposed in Section 2 is implemented into ABAQUS by using the UMAT. The implicit stress integration algorithm and a new expression of the consistent tangent modulus are derived based on the radial return mapping and backward Euler integration methods, respectively.

\subsection{Discretization of Constitutive Equations}

Firstly, it is necessary to discretize constitutive equations by the backward Euler integration method. It is noted that ratcheting behaviors of SA508-3 steel are investigated at different temperatures in this work; however, each temperature is fixed in the process of cyclic loading, and thus the temperature gradient $\dot{T}$ and thermal strain rate $\dot{\varepsilon}^{T}$ can be neglected in following derivations. Considering the interval from a state $n$ to $n+1$, if the terms with $n$ subindexes are known, then the terms with subindexes $n+1$ can be calculated with the following expressions:

$$
\begin{gathered}
\varepsilon_{n+1}=\varepsilon_{n+1}^{e}+\varepsilon_{n+1}^{p} \\
\varepsilon_{n+1}^{p}=\varepsilon_{n}^{p}+\Delta \varepsilon_{n+1}^{p} \\
\boldsymbol{\sigma}_{n+1}=\mathbf{D}(T):\left(\varepsilon_{n+1}-\varepsilon_{n+1}^{p}\right) \\
\Delta \varepsilon_{n+1}^{p}=\sqrt{\frac{3}{2} \Delta p_{n+1} \mathbf{n}_{n+1}} \\
\mathbf{n}_{n+1}=\frac{\mathbf{s}_{n+1}-\boldsymbol{\alpha}_{n+1}}{\left\|\mathbf{s}_{n+1}-\boldsymbol{\alpha}_{n+1}\right\|} \\
F_{y(n+1)}=\sqrt{1.5\left(\mathbf{s}_{n+1}-\alpha_{n+1}\right):\left(\mathbf{s}_{n+1}-\boldsymbol{\alpha}_{n+1}\right)}-Q_{n+1}
\end{gathered}
$$

The discrete evolution rule of kinematic hardening is given by

$$
\boldsymbol{\alpha}_{n+1}=\boldsymbol{\alpha}_{n}+\frac{2}{3} C(p, T) \Delta \varepsilon_{n+1}^{p}-r(T) \boldsymbol{\alpha}_{n+1} \Delta p_{n+1}
$$

The isotropic hardening rule can be discretized as:

$$
\Delta Q_{n+1}(p, T)=\gamma(T)\left(Q_{s a}(T)-Q_{n}(p, T)\right) \Delta p_{n+1}
$$

\subsection{Implicit Stress Integration Algorithm}

It is assumed that the variables at time $t_{n}$ are known, then once the values of $\Delta \varepsilon_{n+1}$ and $\Delta t_{n+1}$ are given, $\sigma_{n+1}$ and the variables at time $t_{n+1}$ can be calculated subsequently.

Assuming the strain increment as elasticity, the trail stress can be obtained as

$$
\boldsymbol{\sigma}_{n+1}^{*}=\mathbf{D}(T):\left(\varepsilon_{n+1}-\varepsilon_{n}^{p}\right)
$$

For the trial stress state, the yield function is given by

$$
F_{y(n+1)}^{*}=\sqrt{\frac{3}{2}\left(\mathbf{s}_{n+1}^{*}-\boldsymbol{\alpha}_{n}\right):\left(\mathbf{s}_{n+1}^{*}-\boldsymbol{\alpha}_{n}\right)}-Q_{n}(p, T)
$$

where $\mathbf{s}_{n+1}^{*}=\boldsymbol{\sigma}_{n+1}^{*}-\frac{1}{3} \operatorname{tr}\left(\boldsymbol{\sigma}_{n+1}^{*}\right)$ I. If $F_{y(n+1)}^{*} \leq 0$, then the trial stress state is accepted as the true stress state. If $F_{y(n+1)}^{*}>0$, then plastic yielding occurs, and then the stress state should be corrected as:

$$
\boldsymbol{\sigma}_{n+1}=\boldsymbol{\sigma}_{n+1}^{*}-\mathbf{D}(T): \Delta \varepsilon_{n+1}^{p}
$$

where $\mathbf{D}(T): \Delta \varepsilon_{n+1}^{p}$ is the plastic corrector. 
It is seen from Equation (21) that $\sigma_{n+1}$ can be obtained once $\Delta \varepsilon_{n+1}^{p}$ is solved. A non-linear scalar equation can be derived to solve this increment. The deviatoric part of Equation (21) can be given as:

$$
\mathbf{s}_{n+1}=\mathbf{s}_{n+1}^{*}-2 G(T) \Delta \varepsilon_{n+1}^{p}
$$

where $G(T)$ denotes the shear modulus.

From Equation (17), $\boldsymbol{\alpha}_{n+1}$ can be rewritten as

$$
\boldsymbol{\alpha}_{n+1}=\theta_{n+1}\left(\boldsymbol{\alpha}_{n}+\frac{2}{3} C_{n+1}(p, T) \Delta \varepsilon_{n+1}^{p}\right)
$$

where $\theta_{n+1}$ is defined by the following form:

$$
\theta_{n+1}=\frac{1}{1+r(T) \Delta p_{n+1}}
$$

Eliminating $\Delta \varepsilon_{n+1}^{p}$ in Equations (22) and (23) with (14) and (15) yields

$$
\mathbf{s}_{n+1}-\boldsymbol{\alpha}_{n+1}=\frac{Q_{n+1}(p, T)\left(\mathbf{s}_{n+1}^{*}-\theta_{n+1} \boldsymbol{\alpha}_{n}\right)}{Q_{n+1}(p, T)+\Delta p_{n+1}\left(3 G(T)+\theta_{n+1} C_{n+1}(p, T)\right)}
$$

Substituting Equation (25) into the yield function Equation (20) gives

$$
\Delta p_{n+1}=\frac{\left[\frac{3}{2}\left(\mathbf{s}_{n+1}^{*}-\theta_{n+1} \boldsymbol{\alpha}_{n}\right):\left(\mathbf{s}_{n+1}^{*}-\theta_{n+1} \boldsymbol{\alpha}_{n}\right)\right]^{\frac{1}{2}}-Q_{n+1}(p, T)}{3 G(T)+\theta_{n+1} C_{n+1}(p, T)}
$$

It is noted that $Q_{n+1}(p, T)$ and $C_{n+1}(p, T)$ are functions related to $\Delta p_{n+1}$ and $T$, the above non-linear scalar equation can be solved by the successive substitution method. The variables at time $t_{n+1}$ will be updated after $\Delta p_{n+1}$ is obtained. The convergence of the successive iteration process had been proved by Kobayashi and Ohno [22]. The scheme of the implicit stress integration process is illustrated in Figure 3.

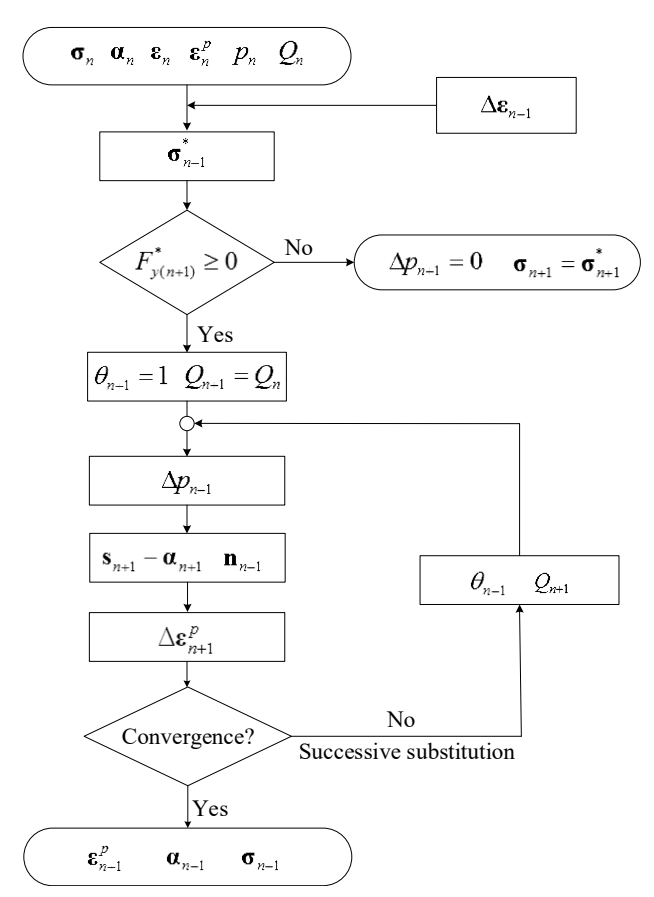

Figure 3. Flow diagram of stress integration algorithm. 


\subsection{Consistent Tangent Modulus}

In the process of finite element implementation, the consistent tangent modulus is necessary to ensure the global equilibrium iteration. In this subsection, a new expression of consistent tangent modulus $\frac{d \Delta \sigma_{n+1}}{d \Delta \varepsilon_{n+1}}$ is derived.

Differentiating Equations (13)-(15) and (18) gives

$$
\begin{gathered}
d \Delta \mathbf{\sigma}_{n+1}=\mathbf{D}(T):\left(d \Delta \varepsilon_{n+1}-d \Delta \varepsilon_{n+1}^{p}\right) \\
d \Delta \varepsilon_{n+1}^{p}=\sqrt{\frac{3}{2}}\left(d \Delta p_{n+1} \mathbf{n}_{n+1}+\Delta p_{n+1} d \mathbf{n}_{n+1}\right) \\
d \mathbf{n}_{n+1}=\sqrt{\frac{3}{2}} \frac{d \Delta \mathbf{s}_{n+1}-d \Delta \boldsymbol{\alpha}_{n+1}}{Q_{n+1}(p, T)}-\frac{\mathbf{n}_{n+1}}{Q_{n+1}(p, T)}\left(\frac{d Q_{n+1}(p, T)}{d p_{n+1}}\right)_{n+1} d \Delta p_{n+1} \\
\mathbf{n}_{n+1}: d \mathbf{n}_{n+1}=0 \\
\frac{d Q_{n+1}(p, T)}{d p_{n+1}}=\gamma\left(Q_{s a}(T)-Q_{n+1}(p, T)\right)
\end{gathered}
$$

It is noted that $\mathbf{D}(T): d \Delta \varepsilon^{p}=2 G(T) d \Delta \varepsilon^{p}$, and taking the deviatoric part of Equation (27) gives

$$
d \Delta \mathbf{s}_{n+1}=2 G(T)\left(\mathbf{I}_{d}: d \Delta \varepsilon_{n+1}-d \Delta \varepsilon_{n+1}^{p}\right)
$$

where $\mathbf{I}_{d}=\mathbf{I}-\frac{1}{3}(1 \otimes 1)$ is the deviatoric operation of a tensor, $\otimes$ stands for operation of tensor product and $\mathbf{I}$ is the fourth order unit tensors.

Combination of Equations (28) and (30) gives

$$
d \Delta p_{n+1}=\sqrt{\frac{2}{3}} \mathbf{n}_{n+1}: d \Delta \varepsilon_{n+1}^{p}
$$

Substituting Equation (29) into Equation (28) gives

$$
d \Delta \varepsilon_{n+1}^{p}=\left[1-\frac{\Delta p_{n+1}}{Q_{n+1}(p, T)}\left(\frac{d Q_{n+1}(p, T)}{d p}\right)_{n+1}\right] \mathbf{n}_{n+1} \otimes \mathbf{n}_{n+1}: d \Delta \varepsilon_{n+1}^{p}+\frac{3}{2} \frac{\Delta p_{n+1}}{Q_{n+1}(p, T)}\left(d \Delta \mathbf{s}_{n+1}-d \Delta \boldsymbol{\alpha}_{n+1}\right)
$$

According to literature [21], it is assumed that the increment of deviatoric back stress has the following differential form:

$$
d \Delta \boldsymbol{\alpha}_{n+1}=\mathbf{H}_{n+1}: d \Delta \varepsilon_{n+1}^{p}
$$

where $\mathbf{H}_{n+1}$ is fourth-order hardening modulus of back stress.

Substituting Equations (35) and (32) into Equation (34) to eliminate $d \Delta \boldsymbol{\alpha}_{n+1}$ and $d \Delta \mathbf{s}_{n+1}$ yields

$$
\mathbf{L}_{n+1}: d \Delta \varepsilon_{n+1}^{p}=2 G(T) \mathbf{I}_{d}: d \Delta \varepsilon_{n+1}
$$

where $\mathbf{L}_{n+1}$ is defined as

$$
\mathbf{L}_{n+1}=2 G(T) \mathbf{I}+\sum_{i=1}^{M} H_{n+1}+\frac{2}{3}\left(\frac{d Q_{n+1}(p, T)}{d p_{n+1}}\right)_{n+1} \mathbf{n}_{n+1} \otimes \mathbf{n}_{n+1}+\frac{2}{3} \frac{Q_{n+1}(p, T)}{\Delta p_{n+1}}\left(\mathbf{I}-\mathbf{n}_{n+1} \otimes \mathbf{n}_{n+1}\right)
$$

Then elimination of $d \Delta \varepsilon_{n+1}^{p}$ in Equation (27) using Equation (36) gives

$$
d \Delta \boldsymbol{\sigma}_{n+1}=\left(\mathbf{D}(T)-4 G(T) \mathbf{L}_{n+1}^{-1}: \mathbf{I}_{d}\right): d \Delta \boldsymbol{\varepsilon}_{n+1}
$$


Therefore, the expression of consistent tangent modulus $\frac{d \Delta \sigma_{n+1}}{d \Delta \varepsilon_{n+1}}$ is derived as

$$
\frac{d \Delta \boldsymbol{\sigma}_{n+1}}{d \Delta \varepsilon_{n+1}}=\mathbf{D}(T)-4 G(T) \mathbf{L}_{n+1}^{-1}: \mathbf{I}_{d}
$$

The main differences between the above derived consistent tangent modulus and that done by Kobayashi and Ohno [22] are reflected in two aspects: the temperature effect considering in the variables and the tensor $\mathbf{H}_{n+1}$ (shown in Equations (35) and (37)) resulting in different formation of $\mathbf{L}_{n+1}$. Though applying different material parameters in the kinematic hardening rule, the general form of Equation (39) seems to be similar to the classical approach of Chaboche model, because the backstress is hided in the tensor $\mathbf{L}_{n+1}$. In order to employ the successive substitution algorithm, it is necessary to obtain the tensor $\mathbf{H}_{n+1}$. Detailed derivation of $\mathbf{H}_{n+1}$ are given below.

For the proposed model, firstly differentiating Equation (23) gives

$$
d \boldsymbol{\alpha}_{n+1}=\frac{d \theta_{n+1}}{\theta_{n+1}} \boldsymbol{\alpha}_{n+1}+\frac{2}{3} \theta_{n+1}\left(C_{n+1}(p, T) d \Delta \varepsilon_{n+1}^{p}+\Delta \varepsilon_{n+1}^{p} d C_{n+1}(p, T)\right)
$$

Besides, differentiating Equation (24) using Equation (33) yields

$$
d \theta_{n+1}=-\sqrt{\frac{2}{3}} \theta_{n+1}^{2} r(T) \mathbf{n}_{n+1}: d \Delta \varepsilon_{n+1}^{p}
$$

Substitution of Equation (40) using Equation (41) becomes

$$
d \boldsymbol{\alpha}_{n+1}=\theta_{n+1}\left\{-\sqrt{\frac{2}{3}} r(T) \boldsymbol{\alpha}_{n+1} \otimes \mathbf{n}_{n+1}+\frac{2}{3}\left[C_{n+1}(p, T) \mathbf{I}+\sqrt{\frac{2}{3}} C_{n+1}(p, T)\left(b+2 c p_{n+1}\right) \Delta \varepsilon_{n+1}^{p} \otimes \mathbf{n}_{n+1}\right]\right\}: d \Delta \varepsilon_{n+1}^{p}
$$

Comparing Equation (35) and Equation (42), $\mathbf{H}_{n+1}$ can be expressed as

$$
\mathbf{H}_{n+1}=\theta_{n+1}\left\{-\sqrt{\frac{2}{3}} r(T) \boldsymbol{\alpha}_{n+1} \otimes \mathbf{n}_{n+1}+\frac{2}{3}\left[C_{n+1}(p, T) \mathbf{I}+\sqrt{\frac{2}{3}} C_{n+1}(p, T)\left(b+2 c p_{n+1}\right) \Delta \varepsilon_{n+1}^{p} \otimes \mathbf{n}_{n+1}\right]\right\}
$$

Then, $\mathbf{L}_{n+1}$ in Equation (37) can be derived by $\mathbf{H}_{n+1}$. Finally, a new expression of consistent tangent modulus $\frac{d \Delta \boldsymbol{\sigma}_{n+1}}{d \Delta \varepsilon_{n+1}}$ can be obtained, as shown in Equation (39).

\section{Verification of Finite Element Implementation}

\subsection{Ratcheting Behaviors in A Material Point}

Stress-controlled cyclic tests are performed to reveal ratcheting behaviors of SA508-3 steel at different temperatures. The experimental temperature is set as $25^{\circ} \mathrm{C}, 150^{\circ} \mathrm{C}$ and $325^{\circ} \mathrm{C}$, the loading condition is set as $50 \pm 450 \mathrm{MPa}$ (mean stress $=50 \mathrm{MPa}$; stress amplitude $=550 \mathrm{MPa}$ ), and the stress rate is prescribed as $100 \mathrm{MPa} / \mathrm{s}$. Corresponding material parameters are listed in Table 1.

Table 1. Material parameters using in the proposed model at different temperatures.

\begin{tabular}{cc}
\hline Temperature $/{ }^{\circ} \mathrm{C}$ & Material Parameters \\
\hline \multirow{2}{*}{25} & $E=2.23 \mathrm{e} 5 \mathrm{MPa}, v=0.3, a=8.52, b=-1.0, c=-319, r=5$, \\
& $Q_{0}=445 \mathrm{MPa}, Q_{1}=447 \mathrm{MPa}, Q_{\mathrm{sa}}=410 \mathrm{MPa}, \gamma=0.6$ \\
\hline \multirow{2}{*}{150} & $E=2.0 \mathrm{e} 5 \mathrm{MPa}, v=0.3, a=8.49, b=-4.29, c=-384.5, r=5$, \\
& $Q_{0}=435 \mathrm{MPa}, Q_{1}=428 \mathrm{MPa}, Q_{\mathrm{sa}}=395.6 \mathrm{MPa}, \gamma=0.6$ \\
\hline \multirow{2}{*}{325} & $E=2.0 \mathrm{e} 5 \mathrm{MPa}, v=0.3, a=8.435, b=0.5, c=-388.5, r=5$, \\
& $Q_{0}=410 \mathrm{MPa}, Q_{1}=412.6 \mathrm{MPa}, Q_{\mathrm{sa}}=413 \mathrm{MPa}, \gamma=0.3$ \\
\hline
\end{tabular}


The experimental stress-strain curves are shown in Figure 4a-c. Only the hysteresis loops in the 1st-5th, 25th and 50th cycles are extracted for more clear observation. It is shown that in the 50th cycle, the strain at $150{ }^{\circ} \mathrm{C}$ is greater than that at $25^{\circ} \mathrm{C}$ when the temperature increases; but it shows a contrary tendency when the temperature is up to $325^{\circ} \mathrm{C}$. This is due to the dynamic strain aging effect, which can improve the material's ability to resist deformation at elevated temperature. Liu et al. [23] also observed similar dynamic strain aging effect of SA508-3 steel when the temperature exceeded $260^{\circ} \mathrm{C}$ at a certain loading rate.

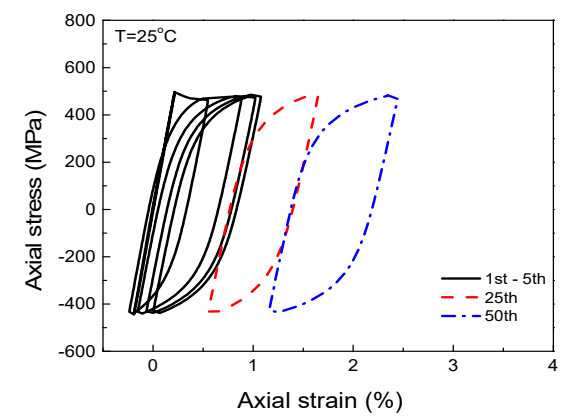

(a)

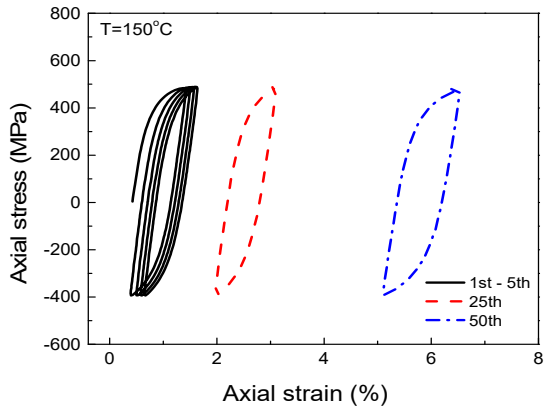

(b)

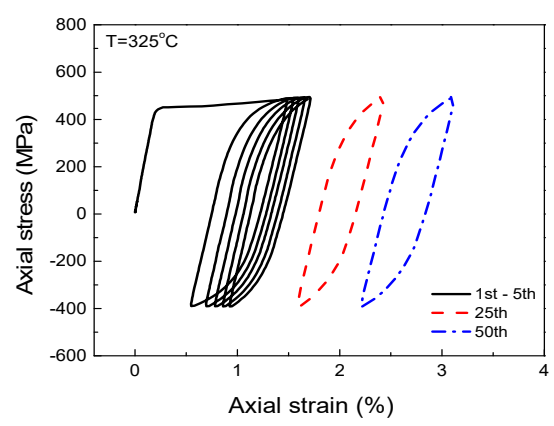

(c)

Figure 4. Experimental stress-strain curves under stress-controlled cycling at (a) $25^{\circ} \mathrm{C}$, (b) $150{ }^{\circ} \mathrm{C}$ and (c) $325^{\circ} \mathrm{C}$.

Simulations in a material point by using the finite element software ABAQUS are performed to verify the implemented model. The ratcheting strain is defined as the averaged value of maximum strain and minimum strain in each cycle. Take the case of $25^{\circ} \mathrm{C}$ as an example, the simulations are made by Chaboche model and the proposed model respectively. The corresponding material parameters for Chaboche model at $25^{\circ} \mathrm{C}$ are: $C_{1}=7 \mathrm{e} 3 \mathrm{MPa}, C_{2}=2.8 \mathrm{e} 3 \mathrm{MPa}, C_{3}=800 \mathrm{MPa}, r_{1}=100$, $r_{2}=25, r_{3}=30$. The simulated results are compared in Figure $5 \mathrm{a}$. It can be seen that the Chaboche model gives a very conservative estimation on the ratcheting strain, while the proposed model gives a relatively good prediction. There is no need to repeat predictions by Chaboche model at $150{ }^{\circ} \mathrm{C}$ and $325^{\circ} \mathrm{C}$, which are also notably over-estimated. Comparisons between experimental and simulated ratcheting strain evolution by the proposed model are demonstrated in Figure 5b. It can be seen that the simulated results match the experimental ones well. Therefore, the implemented model can provide good simulations to ratcheting behaviors of SA508-3 steel. 


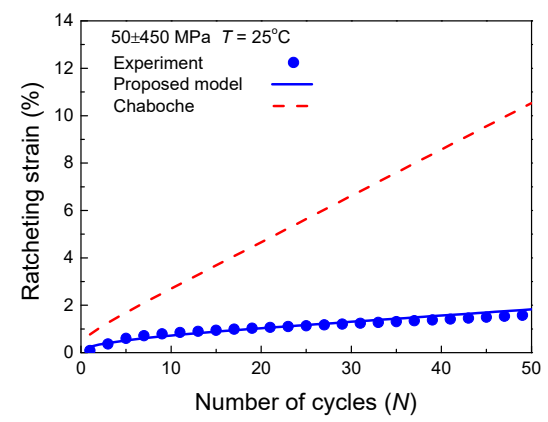

(a)

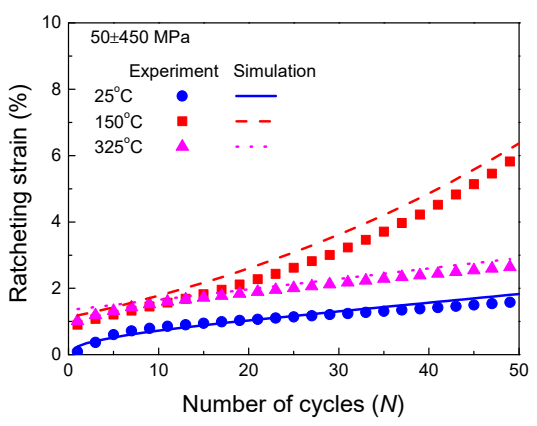

(b)

Figure 5. Comparisons between experimental and simulated ratcheting strain by: (a) Chaboche model and the proposed model at $25^{\circ} \mathrm{C} ;(\mathbf{b})$ the proposed model at different temperatures.

\subsection{Ratcheting Behaviors of Notched Bars}

Uniaxial stress cyclic experiments were performed to investigate ratcheting behaviors of notched bars. The SA508-3 steel was machined into a round notched bar specimen with a section diameter of 10 $\mathrm{mm}$ and a gauge length of $30 \mathrm{~mm}$. The finite element model with two-dimensional (2D) axi-symmetrical four-node element is established in ABAQUS, as shown in Figure 6.

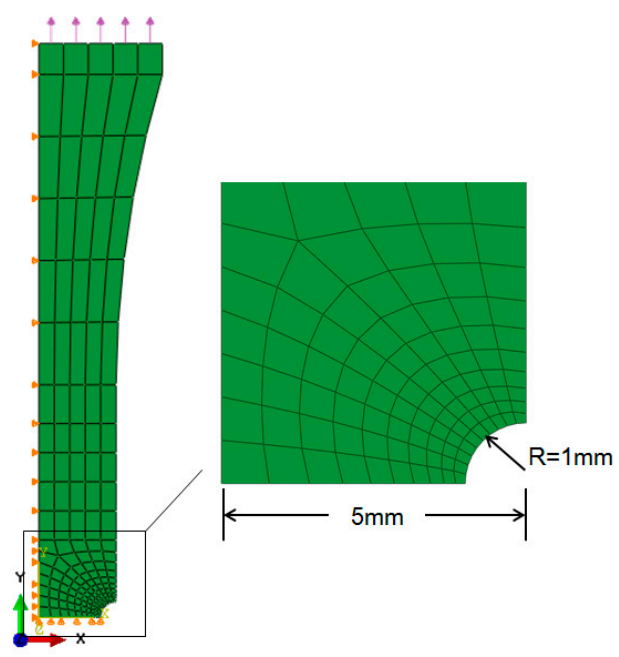

Figure 6. Axi-symmetrical finite element model of the notched bar.

The stress-controlled loading condition is prescribed as $50 \pm 550 \mathrm{MPa}$, and the stress rate is prescribed as $100 \mathrm{MPa} / \mathrm{s}$. Ratcheting strains near the notch center can be obtained from experiments, as shown in Figures 7-9. The notch center enters plastic zone because of the stress concentration. With the increasing of number of cycles, the plastic strain of the notch center accumulates gradually. For the notched bar, the structural strain field is not uniform because of stress concentration effect so that the simulated precision is not as good as that in a material point. The model is modified based on the Chaboche model, though still some efforts should be made to improve it, the conservative prediction has been improved remarkably. Comparisons between experimental and simulated results at $25^{\circ} \mathrm{C}$, $150{ }^{\circ} \mathrm{C}$ and $325^{\circ} \mathrm{C}$ are shown in Figures $7-9$ respectively. It can be seen that the simulated results can give similar evolutions of ratcheting strain to experimental ones. Though the predicted ratcheting strains in the last cycles are higher than those in the initial ones, the proposed model still can give acceptable predictions of ratcheting behaviors of notched bars. 


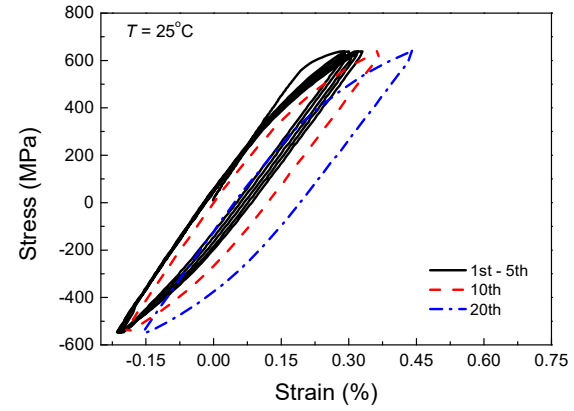

(a)

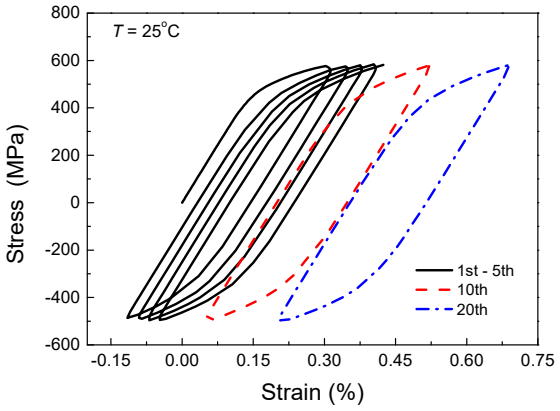

(b)

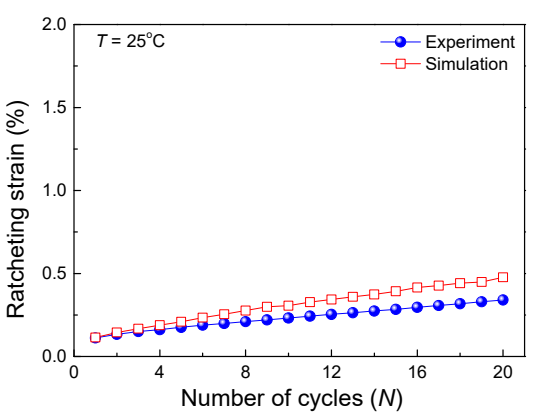

(c)

Figure 7. Results of the notched bar at $25^{\circ} \mathrm{C}$ : (a) experimental stress-strain curves, (b) simulated stress-strain curves and (c) experimental and simulated ratcheting strains versus number of cycles.

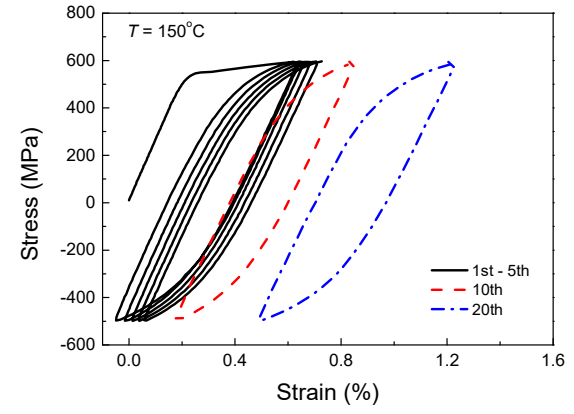

(a)

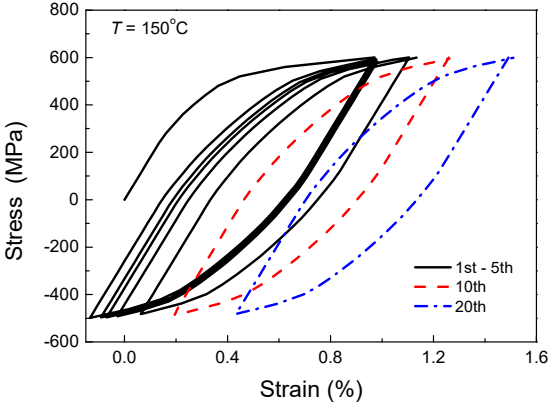

(b)

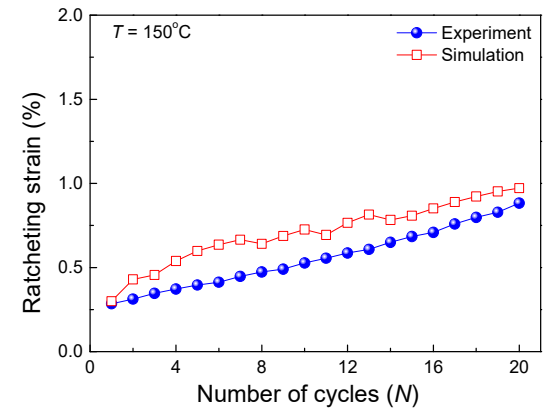

(c)

Figure 8. Results of the notched bar at $150{ }^{\circ} \mathrm{C}$ : (a) experimental stress-strain curves, (b) simulated stress-strain curves and (c) experimental and simulated ratcheting strains versus number of cycles. 


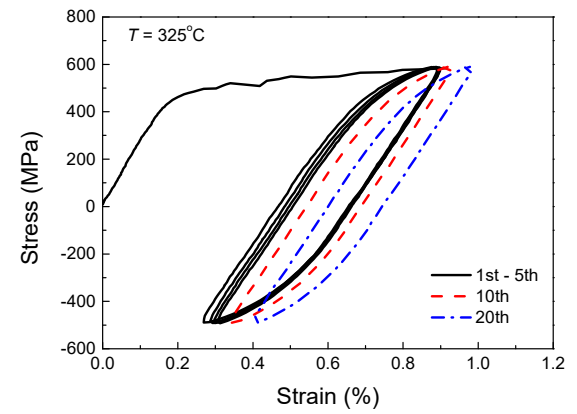

(a)

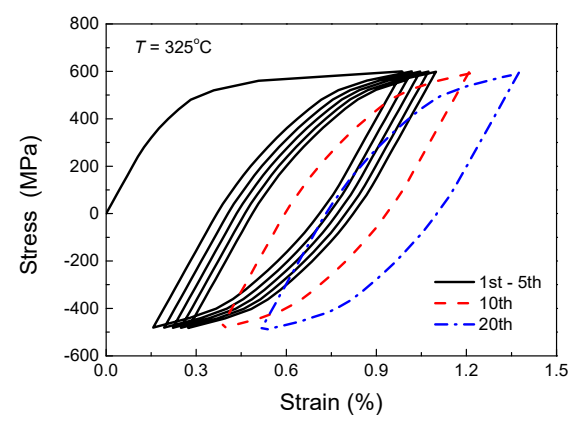

(b)

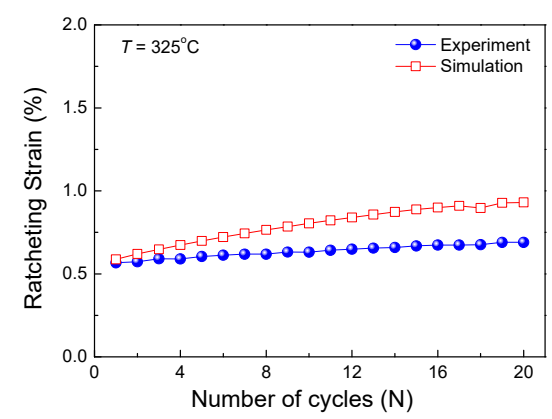

(c)

Figure 9. Results of the notched bar at $325^{\circ} \mathrm{C}$ : (a) experimental stress-strain curves, (b) simulated stress-strain curves and (c) experimental and simulated ratcheting strains versus number of cycles.

\subsection{Ratcheting Behaviors of Plates with A Center Hole}

To accurately simulate the plastic strain accumulation, it is necessary to verify the prediction of strain field distribution. Therefore, the displacement-controlled cyclic tests are performed to obtain the strain field distribution on the surface of plates with a center hole. The thickness of the plate is $2 \mathrm{~mm}$, the gauge length is $10 \mathrm{~mm}$, and the diameter of center hole is $2 \mathrm{~mm}$. A non-contact digital image correlation (DIC) system ARAMIS5M (from GOM mhH Ltd., Brunswick, Germany) is used to measure the full-field strain within the gauged section of specimens. It is difficult to accurately obtain the strain field distribution of plates with a center hole by our DIC device at an elevated temperature since the specimen is heated by a surrounding electric oven and the temperature is also too high to use a transparent temperature controller, which would cut off the field of vision of cameras. Thus, experiments are only carried out at room temperature. The cyclic loading is controlled by displacement with a nominal strain rate of $0.2 \% / \mathrm{s}$ and the nominal strain amplitude is $0.6 \%$. Material parameters used in simulations are shown in Table 1.

It is noted that the tensile strength and fatigue performance of specimens was reported to be affected by scale and manufacturing mechanisms [24]. All tested specimens in this work were machined by the WEDM (Wire Electrical-Discharge Machining, Xinhe machinery processing Ltd., Chengdu, China) technology, which is considered as a valid alternative to milling in tensile specimen manufacturing [25]. Besides, the ratcheting deformation is a type of secondary deformation superimposing on the primary deformation, it is very difficult to predict accurately. In fact, although the proposed model provided more accurate predictions of ratcheting behaviors than the Chaboche model, some differences between predictions and simulations can be observed in all simulations. That is say, comparing to the differences in predictions of ratcheting behaviors, the effects of scale and manufacturing on ratcheting behaviors are relatively small, and thus they are neglected in finite element simulations. A 2D finite element model copying the specimen size is established in ABAQUS, as shown in Figure 10. 


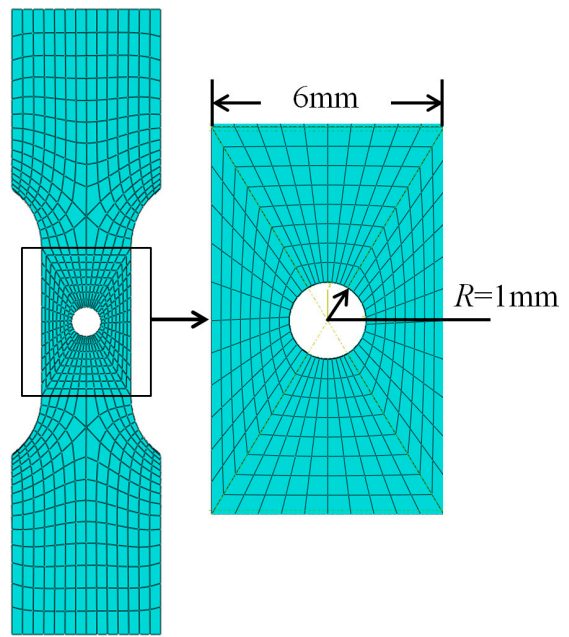

Figure 10. Finite element model of the plate with a center hole.

Comparisons between experimental and simulated results at the 1st, 10th and 20th cycle are shown in Figures 11 and 12. It can be seen that the simulated results of the gauged zone have similar strain field distribution with experimental ones measured by the DIC technology. Besides, the strain value of $\mathrm{Y}$ direction (along the loading direction) on a specific path is extracted to compare the difference between simulated results and measured ones by the DIC technology. The path location is shown in Figure 13a. It can be seen from Figure 13b that the simulated strain along the specific path is acceptable comparing with the experimental results, which means the strain field of the plate with a center hole can be reasonably simulated by the implemented cyclic plastic model.

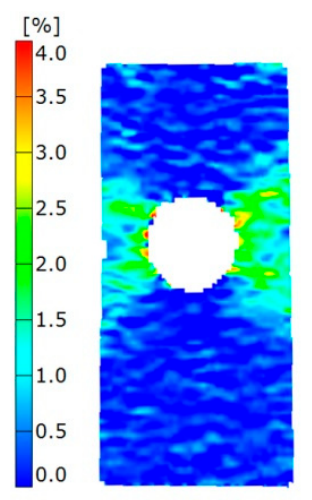

(a)

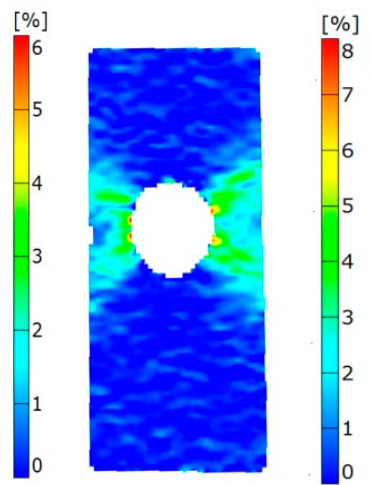

(b)

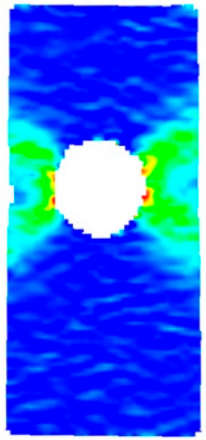

(c)

Figure 11. Experimental strain fields along the loading direction measured by the DIC technology in the (a) 1st cycle, (b) 10th cycle and (c) 20th cycle.

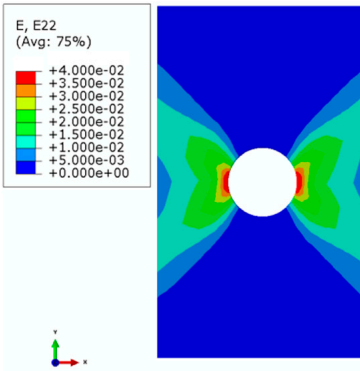

(a)

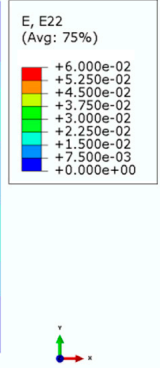

(b)

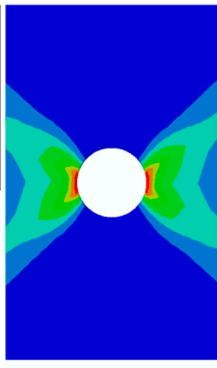

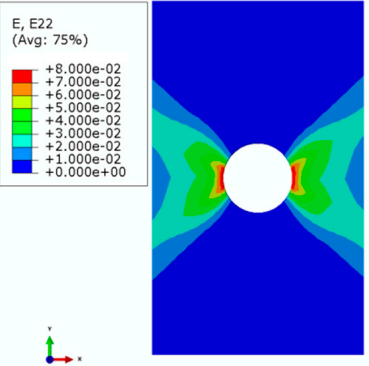

(c)

Figure 12. Simulated strain fields in the (a) 1st cycle, (b) 10th cycle and (c) 20th cycle. 


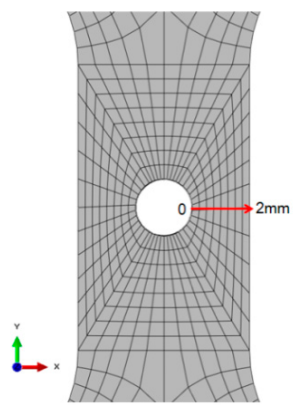

(a)

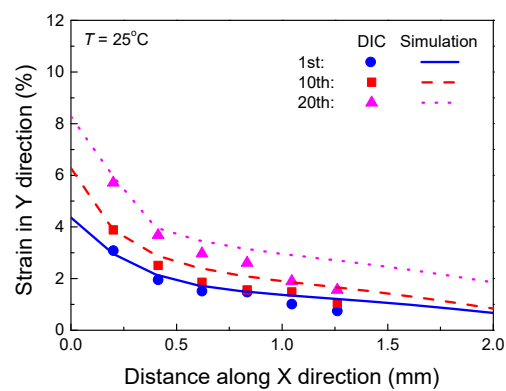

(b)

Figure 13. Comparison between the simulated results and measured ones by the DIC technology: (a) evaluated path location; (b) curves of strain in $\mathrm{Y}$ direction versus distance along $\mathrm{X}$ direction at different temperatures.

\section{Conclusions}

A temperature-dependent cyclic plastic model for SA508-3 steel, considering the nonlinear cyclic softening and kinematic hardening rules, is implemented into a commercial finite element code, i.e., ABAQUS.

- The discrete material parameters in the developed kinematic hardening rule are considered as an exponential function related to the accumulated plastic strain.

- Details of implementation scheme based on the radial return mapping and backward Euler integration methods are presented.

- A new expression of consistent tangent modulus for overall equilibrium iteration is derived.

Then the model is implemented into ABAQUS by using the UMAT. Finite element simulations are carried out to predict ratcheting behaviors of both material point and structures, including notched bars and plates with a center hole. Comparisons between experimental and simulated results show that:

- The implemented temperature-dependent cyclic plastic model can provide reasonable predictions for ratcheting behaviors of structure components of SA508-3 steel at different temperatures.

This work is helpful to evaluate ratcheting behaviors of nuclear reactor structures at different temperatures in further work. It should be mentioned that the working temperature range of the researched material is from room temperature to $350{ }^{\circ} \mathrm{C}$, which has been covered by the present experiments. The adaptability of the proposed model beyond the working temperature range should be validated by performing more experimental researches in further work.

Author Contributions: Methodology, J.T. and J.L.; Supervision, H.X., Y.Y. and Q.K.; Writing—original draft, J.T.; Writing-review \& editing, Q.K.

Funding: This research was funded by the National key research and development program (2016YFB1102601), National Natural Science Foundation of China (11572265), the Excellent Youth Found of Sichuan Province (2017JQ0019) and Exploration Project of Traction Power State Key Laboratory (2017TPLT04) are acknowledged.

Conflicts of Interest: The authors declare no conflict of interest.

\section{References}

1. Bari, S.; Hassan, T. Anatomy of coupled constitutive models for ratcheting simulation. Int. J. Plast. 2000, 16, 381-409. [CrossRef]

2. Bari, S.; Hassan, T. Kinematic hardening rules in uncoupled modeling for multiaxial ratcheting simulation. Int. J. Plast. 2001, 17, 885-905. [CrossRef]

3. Kang, G. Ratchetting: Recent progresses in phenomenon observation, constitutive modeling and application. Int. J. Fatigue 2008, 30, 1448-1472. [CrossRef] 
4. Chen, X.H.; Chen, X.; Yu, D.; Gao, B.J. Recent progresses in experimental investigation and finite element analysis of ratcheting in pressurized piping. Int. J. Press. Vessels Pip. 2013, 101, 113-142. [CrossRef]

5. Chaboche, J.L. Time-independent constitutive theories for cyclic plasticity. Int. J. Plast. 1986, 2, 149-188. [CrossRef]

6. Giannella, V.; Citarella, R.; Fellinger, J.; Esposito, R. LCF assessment on heat shield components of nuclear fusion experiment "Wendelstein 7-X" by critical plane criteria. Procedia Struct. Integr. 2018, 8, 318-331. [CrossRef]

7. Kalnins, A.; Rudolph, J.; Willuweit, A. Using the Nonlinear Kinematic Hardening Material Model of Chaboche for Elastic-Plastic Ratcheting Analysis. J. Press. Vessel Technol. Trans. ASME 2015, 137, 10. [CrossRef]

8. Ohno, N.; Wang, J.D. Kinematic hardening rules with critical state of dynamic recovery, part I: Formulation and basic features for ratchetting behavior. Int. J. Plast. 1993, 9, 375-390. [CrossRef]

9. Ohno, N.; Wang, J.D. Kinematic hardening rules with critical state of dynamic recovery, part II: Application to experiments of ratchetting behavior. Int. J. Plast. 1993, 9, 391-403. [CrossRef]

10. Lin, Y.C.; Liu, Z.H.; Chen, X.M.; Long, Z.L. Cyclic Plasticity Constitutive Model for Uniaxial Ratcheting Behavior of AZ31B Magnesium Alloy. J. Mater. Eng. Perform. 2015, 24, 1820-1833. [CrossRef]

11. Agius, D.; Kourousis, K.I.; Wallbrink, C.; Hu, W.; Wang, C.H.; Dafalias, Y.F. Aluminum Alloy 7075 Ratcheting and Plastic Shakedown Evaluation with the Multiplicative Armstrong-Frederick Model. AIAA J. 2017, 55, 2461-2470. [CrossRef]

12. Chen, W.; Kitamura, T.; Feng, M. Creep and fatigue behavior of 316L stainless steel at room temperature: Experiments and a revisit of a unified viscoplasticity model. Int. J. Fatigue 2018, 112, 70-77. [CrossRef]

13. Blaizot, J.; Chaise, T.; Nélias, D.; Perez, M.; Cazottes, S.; Chaudet, P. Constitutive model for nickel alloy 690 ( Inconel 690$)$ at various strain rates and temperatures. Int. J. Plast. 2016, 80, 139-153. [CrossRef]

14. Kang, G.; Liu, Y. Uniaxial ratchetting and low-cycle fatigue failure of the steel with cyclic stabilizing or softening feature. Mater. Sci. Eng. A 2008, 472, 258-268. [CrossRef]

15. Yaguchi, M.; Takahashi, Y. Ratchetting of viscoplastic material with cyclic softening, part 2: Application of constitutive models. Int. J. Plast. 2005, 21, 835-860. [CrossRef]

16. Abdel-Karim, M.; Ohno, N. Kinematic hardening model suitable for ratchetting with steady-state. Int. J. Plast. 2000, 16, 225-240. [CrossRef]

17. Halama, R.; Markopoulos, A.; Jančo, R.; Bartecký, M. Implementation of MAKOC cyclic plasticity model with memory. Adv. Eng. Softw. 2017, 113, 34-46. [CrossRef]

18. Bonora, N.; Majzoobi, G.H.; Khademi, E. Numerical implementation of a new coupled cyclic plasticity and continum damage model. Comput. Mater. Sci. 2014, 81, 538-547. [CrossRef]

19. Dalla Palma, M. Modelling of cyclic plasticity for austenitic stainless steels 304L, 316L, 316L(N)-IG. Fusion Eng. Des. 2016, 109-111, 20-25. [CrossRef]

20. Agius, D.; Kajtaz, M.; Kourousis, K.I.; Wallbrink, C.; Wang, C.H.; Hu, W.; Silva, J. Sensitivity and optimisation of the Chaboche plasticity model parameters in strain-life fatigue predictions. Mater. Des. 2017, 118, 107-121. [CrossRef]

21. Frederick, C.O.; Armstrong, P.J. A Mathematical Representation of the Multiaxial Bauscinger Effect. Mater. High Temp. 1998, 24, 1-26.

22. Kobayashi, M.; Ohno, N. Implementation of cyclic plasticity models based on a general form of kinematic hardening. Int. J. Numer. Methods Eng. 2002, 53, 2217-2238. [CrossRef]

23. Liu, J.; Wang, L.; Yang, Y.; Cui, J. Fracture toughness and behavior of SA508-III steel under different loading rates. Heat Treat. Met. 2017, 42, 42-46.

24. Tomlinson, S.M.; Lopez-Anido, R.A. Scale and manufacturing effects on tensile strength of marine grade sandwich composite panel joints. J. Sandwich Struct. Mater. 2018. [CrossRef]

25. Krahmer, D.M.; Polvorosa, R.; López de Lacalle, L.N.; Alonso-Pinillos, U.; Abate, G.; Riu, F. Alternatives for Specimen Manufacturing in Tensile Testing of Steel Plates. Exp. Tech. 2016, 40, 1555-1565. [CrossRef]

(C) 2018 by the authors. Licensee MDPI, Basel, Switzerland. This article is an open access article distributed under the terms and conditions of the Creative Commons Attribution (CC BY) license (http:/ / creativecommons.org/licenses/by/4.0/). 